\title{
Low Threshold Voltages Electrochemically Drive Gold Migration in Halide Perovskite Devices
}

Ross A. Kerner ${ }^{a, b}$, Lianfeng Zhao ${ }^{a}$, Steven P. Harvey ${ }^{b}$, Joseph J. Berry ${ }^{b}$, Jeffrey Schwartz, ${ }^{c}$ and Barry P. Rand $^{\mathrm{a}, \mathrm{d}}$

a. Department of Electrical Engineering, Princeton University, Princeton, New Jersey 08544, United States

b. National Renewable Energy Laboratory, Golden, Colorado 80401, United States

c. Department of Chemistry, Princeton University, Princeton, New Jersey 08544, United States

d. Andlinger Center for Energy and the Environment, Princeton University, Princeton, New Jersey 08544, United States

\section{Supporting Information}

Thirteen figures; general experimental methods; discussion of Supporting Information Figures S10 and S11.

\section{Experimental Methods}

Methylammonium iodide, $\mathrm{Pbl}_{2}$, and spiro-MeOTAD were purchased from GreatCell solar, $\mathrm{TCl}$, and Lumtec, respectively. N,N-dimethylformamide (DMF), dimethylsulfoxide (DMSO), methyl acetate (MeOAc) and chlorobenzene (all anhydrous) were purchased from Sigma Aldrich. Devices were fabricated on patterned FTO substrates (TEC 15, purchased from Xin Yan Technologies LTD) that were cleaned by detergent with a toothbrush followed by sonication in deionized water, followed by acetone, and then isopropanol. Solutions of $\mathrm{MAPbl}_{3}$ (stoichiometric) were made $1.2 \mathrm{mM} / \mathrm{mL}$ in a DMF:DMSO solvent mixture (4:1 ratio by volume) 1-3 $\mathrm{h}$ before spin coating. Immediately prior to spin coating, substrates were cleaned by UV-ozone for $15 \mathrm{~min}$. Perovskite films were spin coated in a $\mathrm{N}_{2}$ glovebox/flowbox using the recipe: $1000 \mathrm{rpm}$ for $10 \mathrm{~s}$ then ramped to $6000 \mathrm{rpm}(2000 \mathrm{rpm} / \mathrm{s})$ for $20 \mathrm{~s}$. Approximately $250 \mu \mathrm{L}$ of MeOAc was pipetted onto the rotating substrate with $5 \mathrm{~s}$ left in the spin cycle. The films were then annealed at $100{ }^{\circ} \mathrm{C}$ for $25 \mathrm{~min}$. MAPbl 3 layers processed this way typically display $15-18 \%$ power conversion efficiency with poly-triarylamine (PTAA)/poly[(9,9-bis(3'-((N,N -dimethyl)- $\mathrm{N}$-ethylammonium)- propyl)-2,7fluorene)-alt -2,7-(9,9-dioctylfluorene)] (PFN-Br) hole transport layer and $\mathrm{C}_{60} /$ bathocuproine/Ag electron transport layer/electrode architecture. Spiro-MeOTAD was dissolved in chlorobenzene at $85 \mathrm{mg} / \mathrm{mL}$ concentration and spin coated at $2500 \mathrm{rpm}$. Shadow mask patterned Au electrodes (active device area $\approx$ $0.11 \mathrm{~cm}^{2}$ ) were thermally evaporated (base and deposition pressure $\sim 8 \times 10^{-7} \mathrm{Torr}$ ) at $0.5 \mathrm{~nm} / \mathrm{s}$ for the first $10 \mathrm{~nm}$ and then $2 \mathrm{~nm} / \mathrm{s}$ for the remaining $55 \mathrm{~nm}$ (total of $65 \mathrm{~nm}$ ).

Current-voltage characterization was performed in the dark in a $\mathrm{N}_{2}$ glovebox ( $30 \mathrm{ppm} \mathrm{\textrm {O } _ { 2 }}$ ) with a Keithley DMM6500. STEM lamella samples of the devices were prepared by an FEI Helios DualBeam microscope. A Pt protection layer is deposited on top of the Au electrodes to avoid ion damage during TEM sample preparation. STEM images and EDX measurements were carried out in an FEI Talos (S)TEM at $200 \mathrm{kV}$. An ION-TOF TOF-SIMS V Time of Flight SIMS (TOF-SIMS) spectrometer was utilized for depth profiling of the perovskite device utilizing methods covered in detail in previous reports. ${ }^{1-3}$ Analysis was completed utilizing a 3-lens $30 \mathrm{kV}$ BiMn primary ion gun. High mass resolution depth profiles were completed with a $30 \mathrm{keV} \mathrm{Bi}{ }^{3+}$ primary ion beam, ( $0.8 \mathrm{pA}$ pulsed beam current), a $50 \times 50 \mu \mathrm{m}$ area was analyzed with a 128:128 primary beam raster. The primary ion beam dose density was kept below $1 \times 10^{12}$ ions $\mathrm{cm}^{-2}$ to remain under the static-sims limit. Sputter depth profiling was accomplished with 1 
$\mathrm{kV}$ cesium ion beam ( $7 \mathrm{nA}$ sputter current) with a raster of $150 \times 150 \mu \mathrm{m}$. The Au electrodes were delaminated with tape prior to TOF-SIMS measurements and only uniform areas (visible in the integrated optical microscope) were depth profiled. 

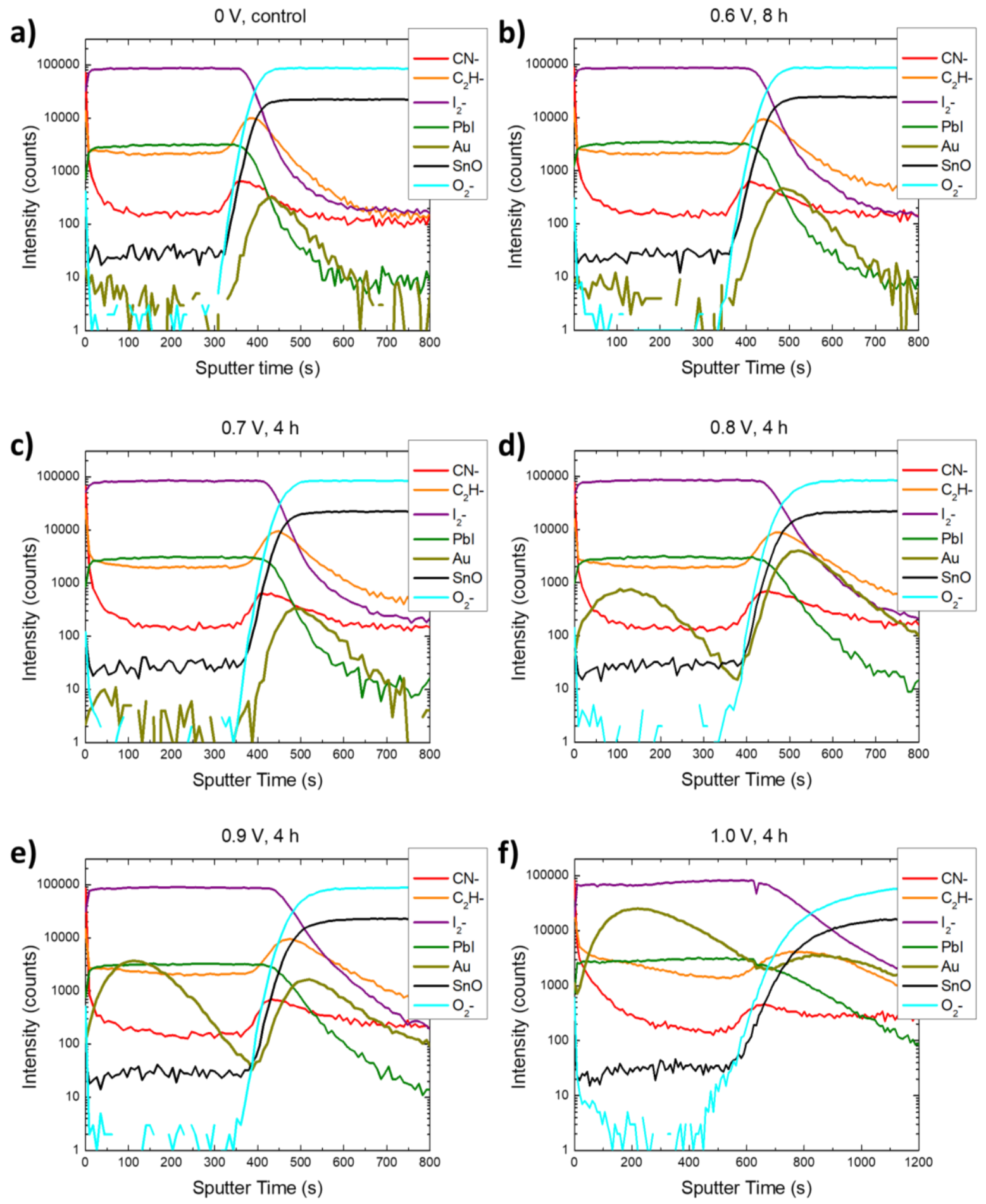

Supporting Information Figure S1. Additional ion fragment TOF-SIMS profiles of an FTO/MAPbl $3 / \mathrm{Au}$ device with pixels biased at (a) $0 \mathrm{~V}$, (b) $0.6 \mathrm{~V}$, (c) $0.7 \mathrm{~V}$, (d) $0.8 \mathrm{~V}$, (e) $0.9 \mathrm{~V}$, and (f) $1.0 \mathrm{~V}$. 


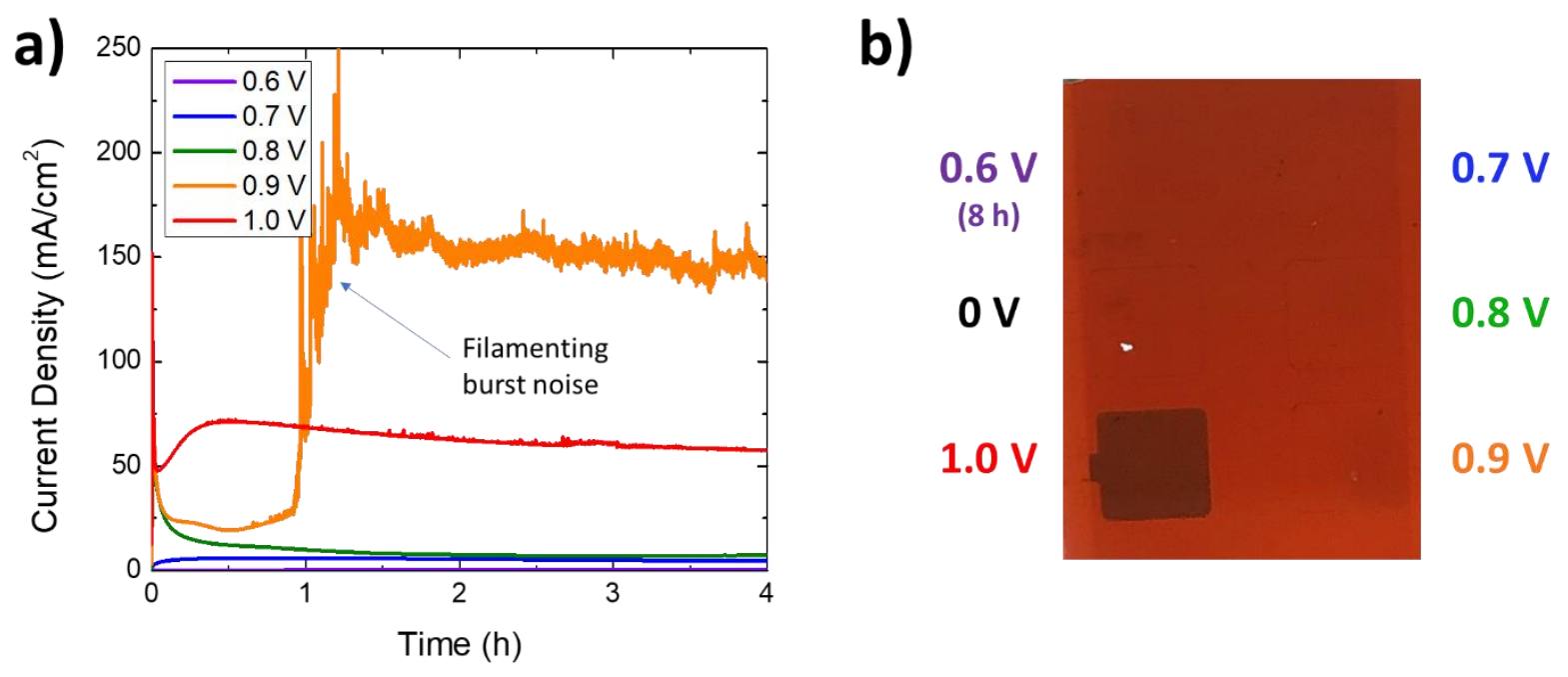

Supporting Information Figure S2. Current density versus time (a) and photograph (b) of the $\mathrm{FTO} / \mathrm{MAPb}_{3} / \mathrm{Au}$ device used to obtain the SIMS data in Figure 1a of the main text (Au electrode removed). The pixel biased at $1.0 \mathrm{~V}$ for $4 \mathrm{~h}$ with the largest Au content displayed significant darkening due to the build-up of colloidal Au in the perovskite bulk. Filamenting as, indicated above, was observed to occur for many pixels. However, filamenting did not seem to affect the general Au migration characteristics. These defects may be locally weak areas, such as very small pinholes or thin areas, that dominate the $J$ - $V$ but do not influence the majority of the pixel area.

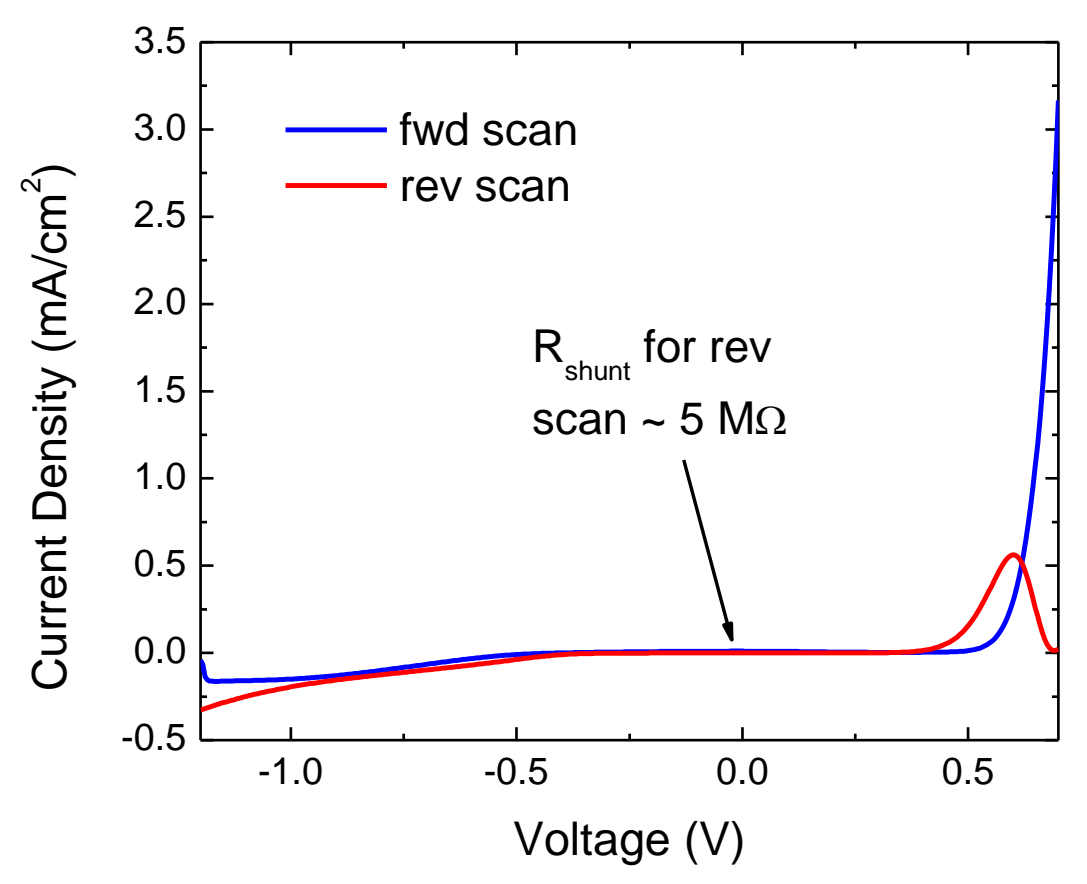

Supporting Information Figure S3. Initial current density versus voltage $(J-V)$ of the $\mathrm{FTO} / \mathrm{MAPbl}_{3} / \mathrm{Au}$ device biased at $0.8 \mathrm{~V}$ in Supporting Information Figure S2. The scan rate was approximately $110 \mathrm{mV} / \mathrm{s}$. 


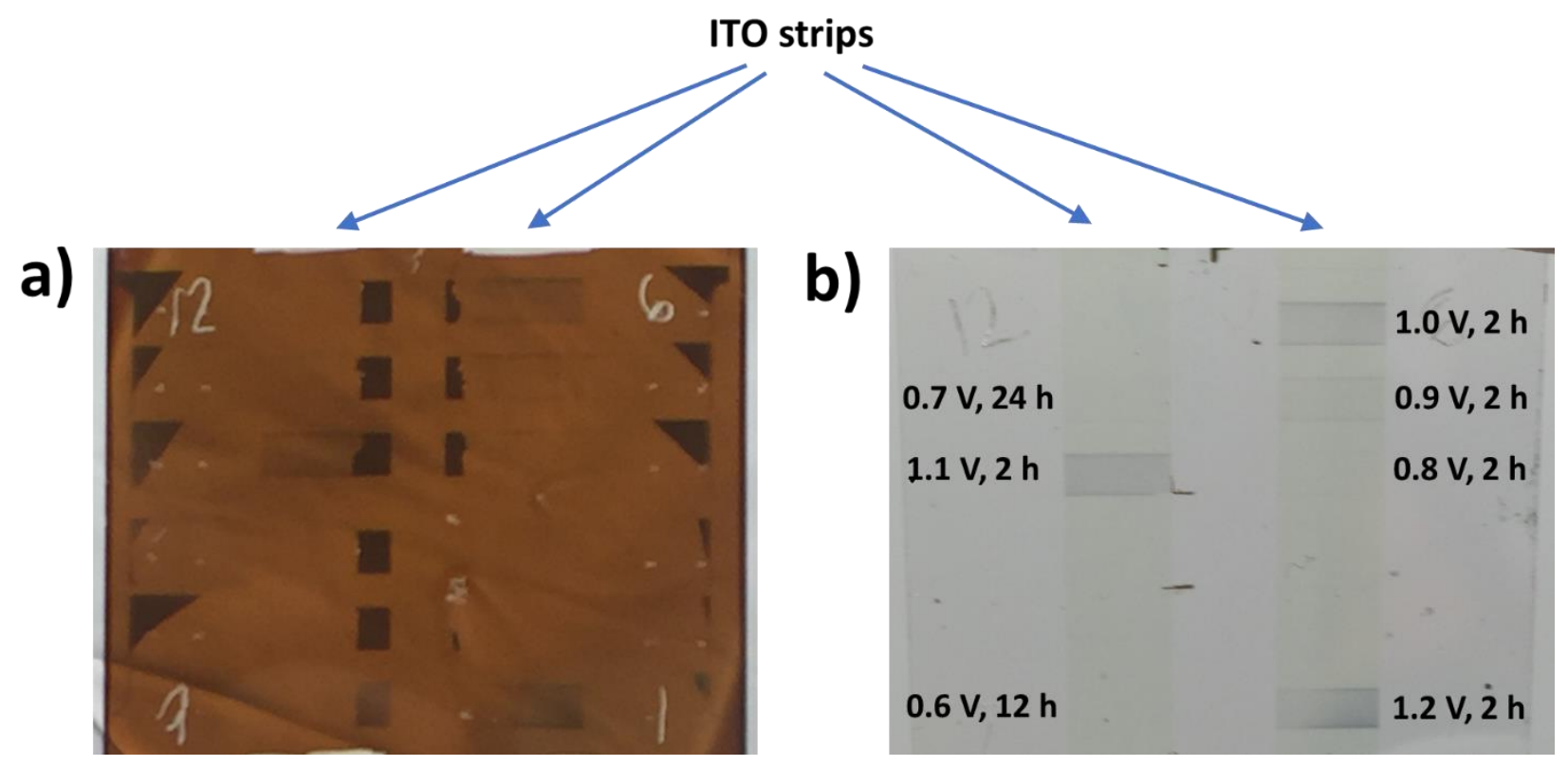

Supporting Information Figure S4. Photographs of an indium tin oxide (ITO, UV-ozone cleaned)/MAPbl $3 / \mathrm{Au}$ device with pixels biased at the indicated voltages. The $\mathrm{MAPbl}_{3}$ in this device was $180 \mathrm{~nm}$ thick, and was processed from N,N-dimethylformamide (DMF) alone (without DMSO) at $0.75 \mathrm{M}$ concentration. Identical thresholds using $\mathrm{MAPbl}_{3}$ prepared in DMF at $1.2 \mathrm{M}$, which was $>300 \mathrm{~nm}$ thick. The Au was removed with tape (a), and the perovskite was removed by DMF (b), showing that significant $\mathrm{Au}$ accumulated at the ITO, not $\mathrm{Pb}^{0}$, as verified by $\mathrm{X}$-ray photoemission spectroscopy (XPS). The close similarity in voltage threshold measured here as that for FTO and a thicker layer of $\mathrm{MAPbl}_{3}$ that processed from a different solvent indicates that Au migration is dictated by electrochemistry at the perovskite/Au interface, and is not specific to crystallinity/morphology. 
a)

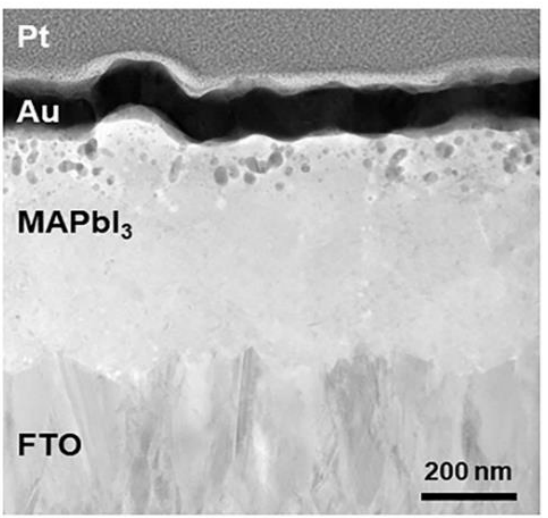

c)

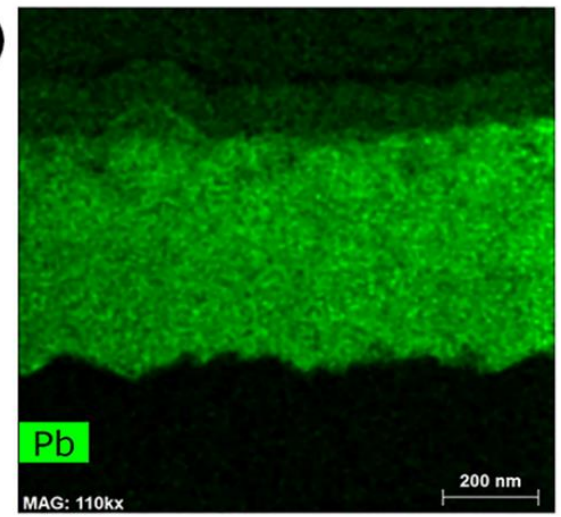

b)

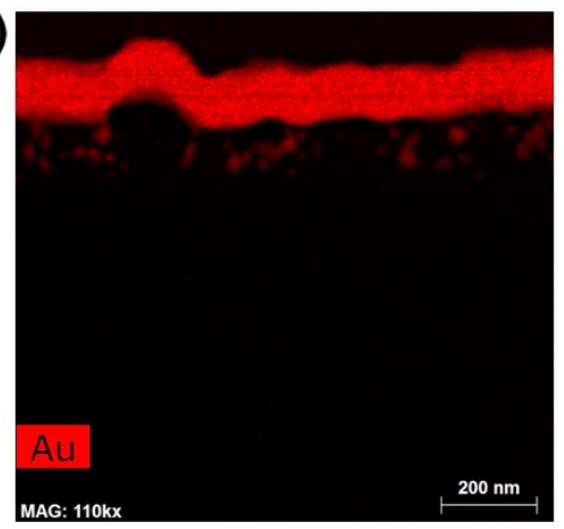

d)

Sn

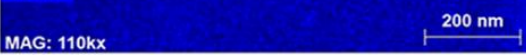

Supporting Information Figure S5. STEM/EDX elemental mapping of an FTO/MAPbl 3 /Au device biased at $0.9 \mathrm{~V}$ for $6 \mathrm{~h}$. (a) Bright-field STEM image, and elemental spatial distributions of (b) Au, (c) Pb, and (d) Sn.

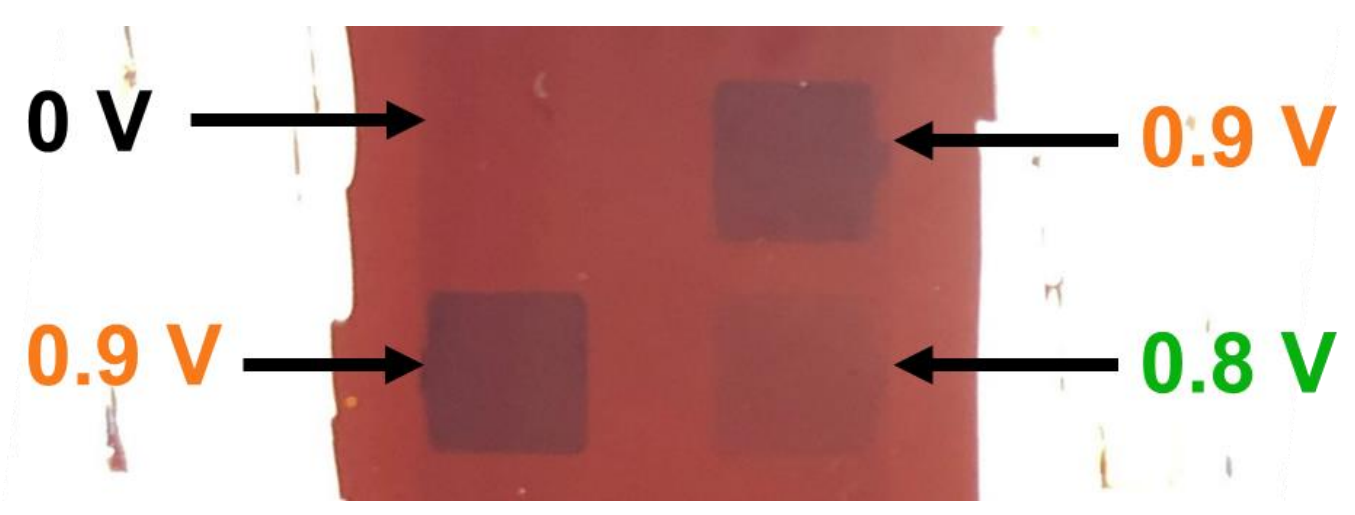

Supporting Information Figure S6. Photograph of a different FTO/MAPbl 3 /Au device with pixels biased at the indicated voltage for $12 \mathrm{~h}$ after delaminating the Au electrodes. At longer biasing times, visible Au accumulates in pixels biased nearer the threshold voltage of $0.8 \mathrm{~V}$ as well. 

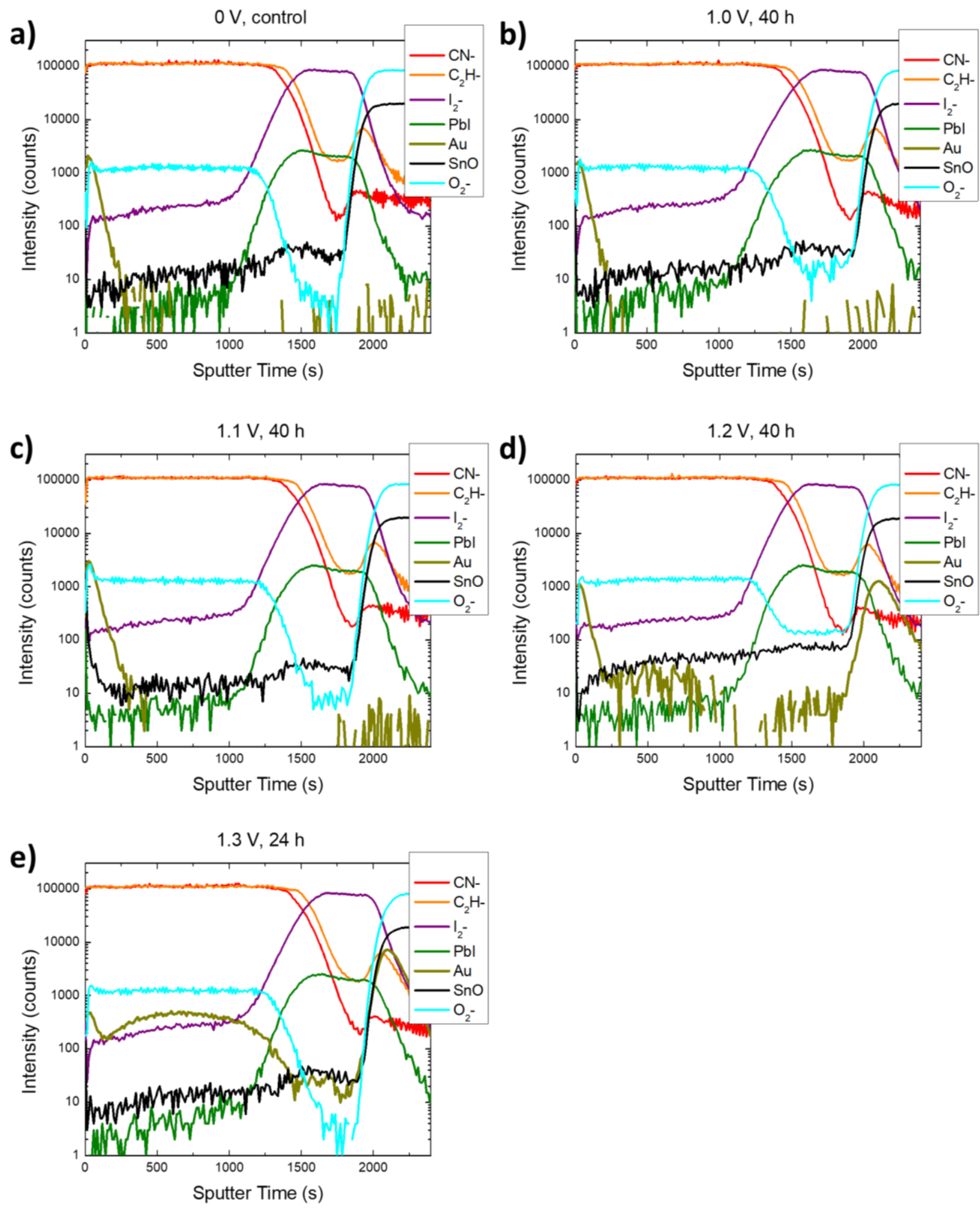

Supporting Information Figure S7. Additional ion fragment TOF-SIMS profiles of an FTO/MAPbl 3 /(undoped) spiro-MeOTAD/Au device with pixels biased at (a) $0 \mathrm{~V}$, (b) $1.0 \mathrm{~V}$, (c) $1.1 \mathrm{~V}$, (d) 1.2 $\mathrm{V}$, and (e) $1.3 \mathrm{~V}$. 


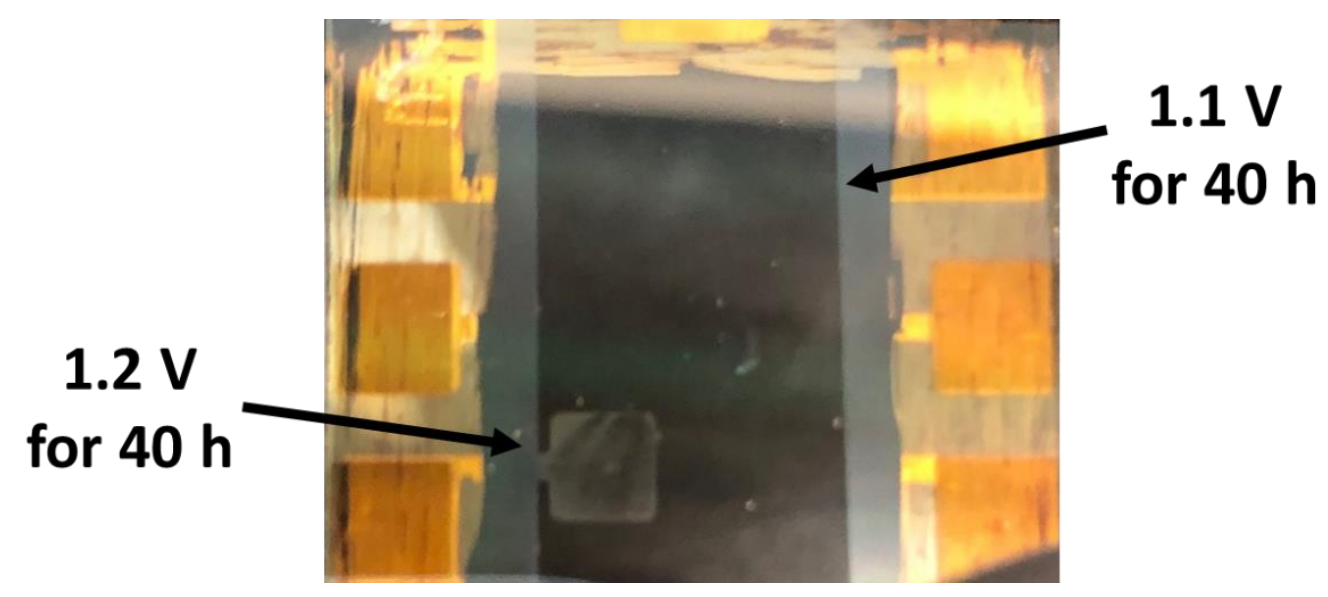

Supporting Information Figure S8. Photograph of an FTO/MAPbl 3 /spiro-MeOTAD (undoped)/Au device through the glass/FTO comparing pixels biased at $1.1 \mathrm{~V}$ and $1.2 \mathrm{~V}$ for $40 \mathrm{~h}$. At a threshold of $1.2 \mathrm{~V}$, corresponding to the Au migration threshold, the FTO/MAPbl 3 interface becomes discolored/degraded. The magnitude of Au accumulation was not substantial enough to discolor this interface (revealed by removing $\mathrm{MAPbl}_{3}$ with $\mathrm{DMSO}$ ). Thus, we believe the discoloration is degradation of $\mathrm{MAPbl}_{3}$ to $\mathrm{Pbl}_{2}$ as $\mathrm{MA}^{+}$ cations are deprotonated at this interface. 


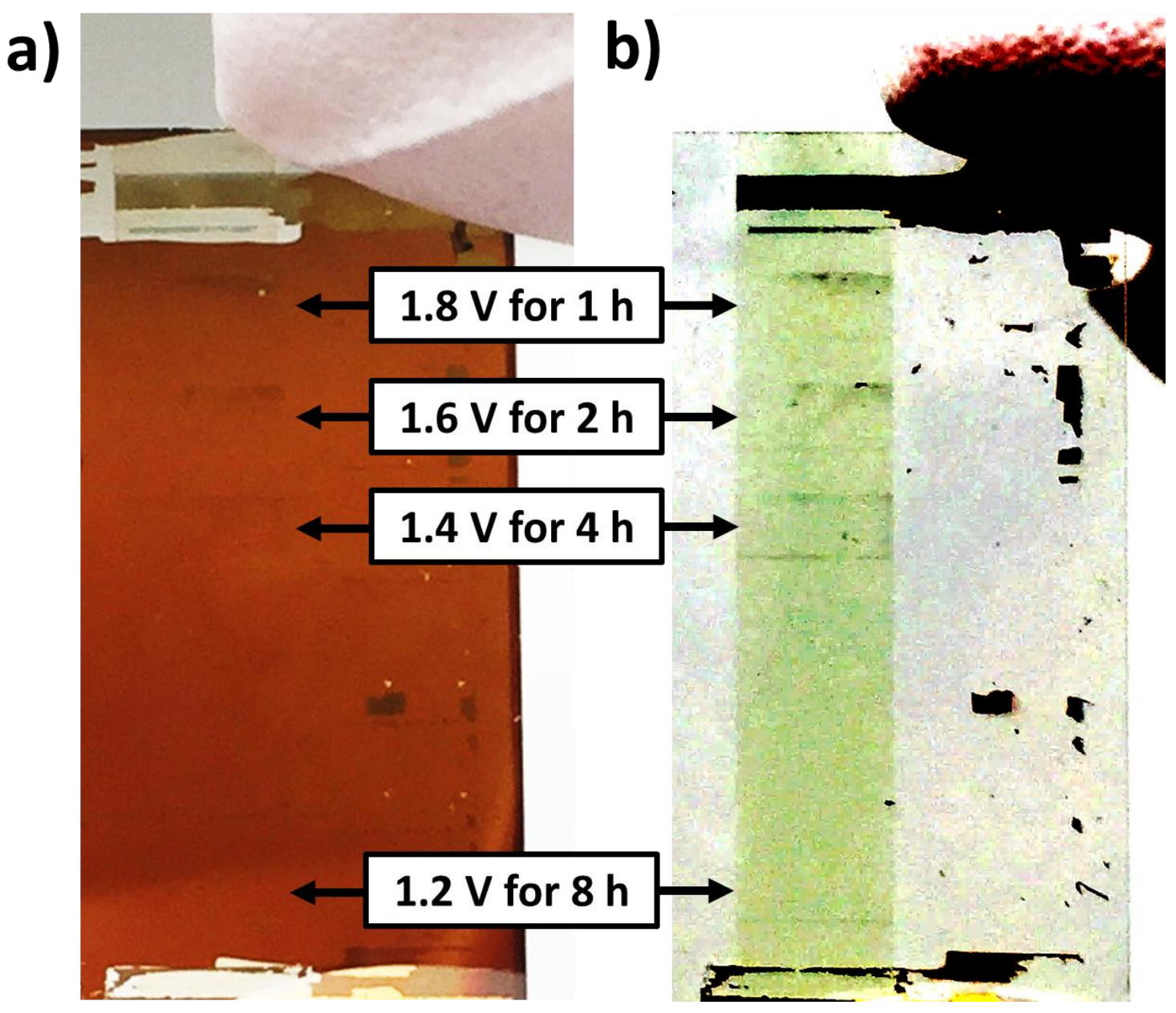

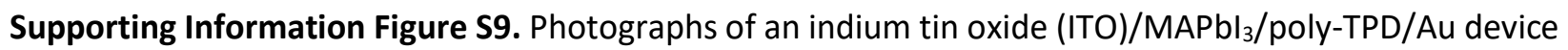
with pixels biased at the indicated voltages after (a) delaminating the Au electrodes, and (b) subsequently washing away the $\mathrm{MAPbl}_{3}$. The $\mathrm{MAPbl}_{3}$ in this device was $180 \mathrm{~nm}$ thick and was processed from DMF (without DMSO) at $0.75 \mathrm{M}$ concentration. Poly-TPD was spin coated from $6 \mathrm{mg} / \mathrm{mL}$ in chlorobenzene, forming a 40-50 $\mathrm{nm}$ thick layer. Contrast and brightness of the photographs were adjusted to better resolve the small amount of Au accumulation in the $1.2 \mathrm{~V}$ pixel. 


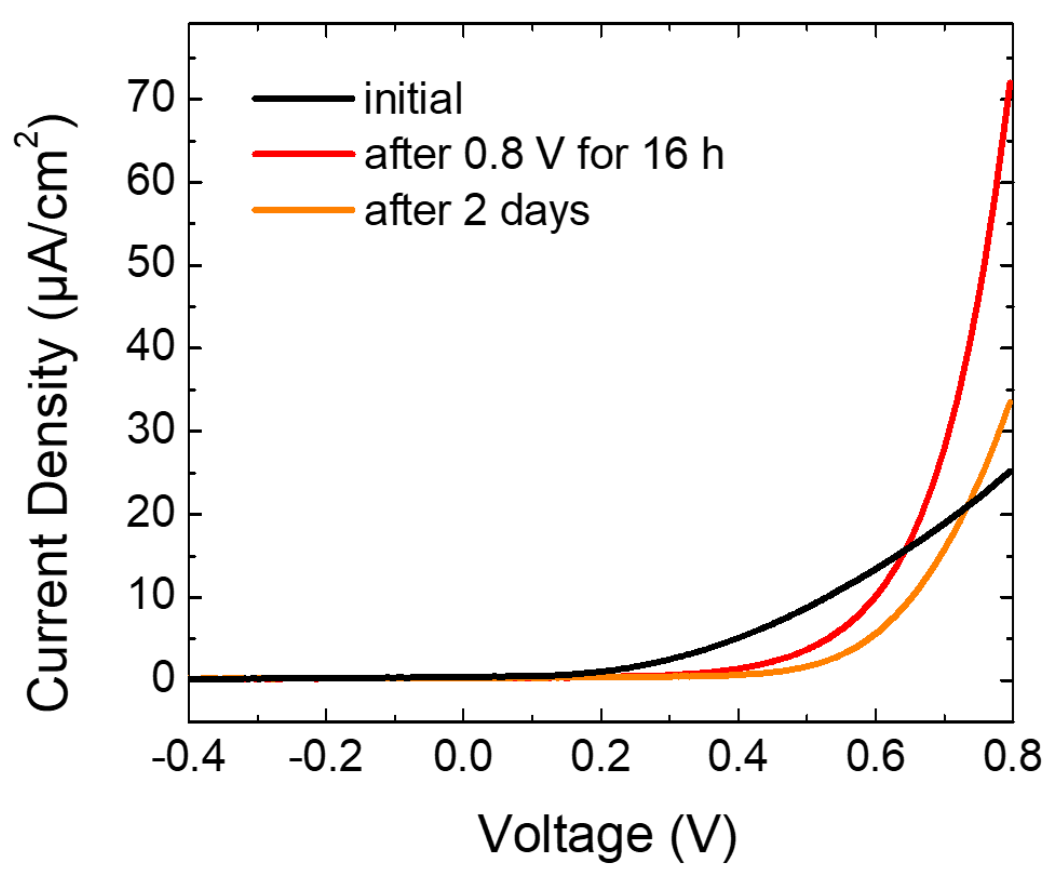

Supporting Information Figure S10. Current density-voltage $(J-V)$ measurements (scan rate $\approx 110 \mathrm{mV} / \mathrm{s}$ ) of an FTO/MAPbl 3 /spiro-MeOTAD (undoped)/Au device pixel before and after biasing at $0.8 \mathrm{~V}$ for $16 \mathrm{~h}$. The initial state of the device appears to be limited by series resistance plausibly due to the undoped spiro-MeOTAD hole transport layer. Biasing at $0.8 \mathrm{~V}$, much lower than the voltages needed to induce Au diffusion, is sufficient to dope the spiro-MeOTAD reducing series resistance. The doping changes were quasi-irreversible on the time scale of days stored in an $\mathrm{N}_{2}$ glovebox (orange curve).
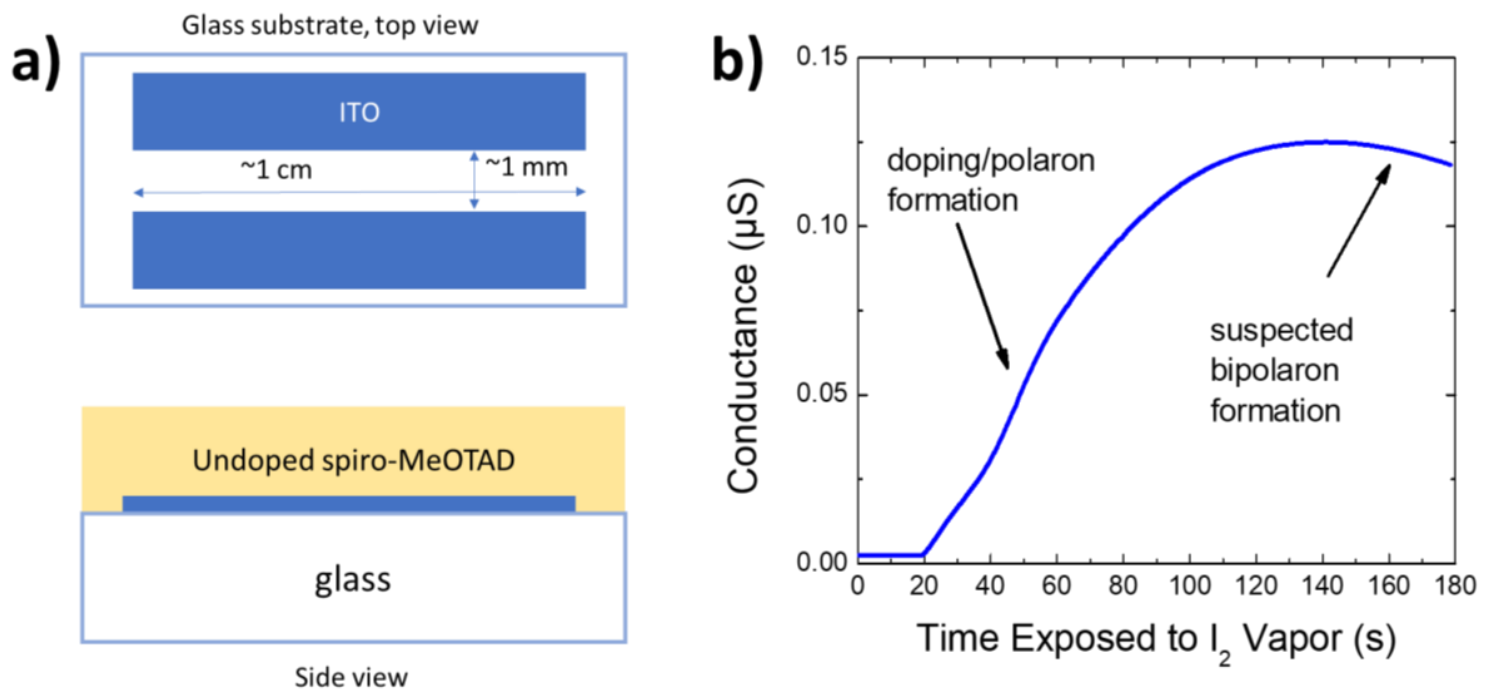

Supporting Information Figure S11. (a) Schematic of the device structure which was used to obtain the $J$ - $t$ data in (b) upon exposure to $I_{2}$ vapor. In studies on other HTMs, the dip in conductivity is attributed to carrier mobility loss as singly oxidized units are further oxidized to bipolarons. ${ }^{4}$ 


\section{Discussion of Supporting Information Figures S10, S11, and spiro-MeOTAD Doping}

Spiro-MeOTAD oxidation by $\mathrm{I}_{2}$ is demonstrated by lateral thin film devices showing strong and reversible doping by $I_{2}$ vapor (Figure S11) exposure which tells us that the speciation of iodide or other ions in the organic will depend on organic semiconductor redox properties. Doping of poly-3octylthiophene by $I_{2}$ vapor results in triiodide (as opposed to iodide) despite a large excess of organic ( 0.054 iodine atoms per monomer unit) and we assume that the speciation is also triiodide for other HTMs with similar frontier orbital energies. ${ }^{5}$ Pentacene thin films doped by $I_{2}$ vapor similarly results in triiodide inclusion. ${ }^{6}$ The decrease in conductance at long times is attributed to bipolaron formation at high oxidation/doping levels. ${ }^{4}$ Thus, we conclude that the preferred iodine speciation in the spiro-MeOTAD layer is also triiodide.

In particular, there are two features that indicate electrochemical doping of the spiro-MeOTAD HTM. The $J$ - $t$ data in Figure $\mathbf{3 a}$ and $\mathbf{3 b}$ display a turn-on transient ( 20-30 $\mathrm{min}$ ) that is independent of applied voltage. The increase in current density at short times is attributed to increasing conductivity of the spiro-MeOTAD layer as iodide from the perovskite is oxidized and can enter the HTM. Voltage independence of the transient suggests that doping of the Spiro-MeOTAD layer is limited by diffusion of triiodide as depicted in Figure 3c. Current density-voltage $(J-V)$ data for a device as a function of characterization history are shown in Figure S10. Initially, the J-V curves for HTM containing devices resemble diodes with low turn-on voltage and high series resistance. This is logical given both the $\mathrm{MAPbl}_{3}$ and spiro-MeOTAD layers should be near intrinsic as-fabricated with small energy barriers (excluding contacts) and conductivities. However, after biasing at $0.8 \mathrm{~V}$ for $16 \mathrm{~h}$, the turn-on voltage significantly increases, and the series resistance no longer limits current in forward bias. The spiro-MeOTAD no longer limiting current implies that the doping is sufficiently high that little electric field is dropped across the spiro-MeOTAD layer. These effects are quasi-irreversible. The species that induce doping prior to $\mathrm{MAPbl}_{3}$ bulk degradation at voltages $<1.2 \mathrm{~V}$ may source from a small amount of reactive defects, such as the defects speculated to spontaneously mobilize some $\mathrm{Au}$ in the unbiased $\mathrm{FTO} / \mathrm{MAPbl}_{3} / \mathrm{Au}$ device in Figure $1 \mathrm{a}$, or loosely bound ions within the $\mathrm{MAPbl}_{3}$, such as a small amount of absorbed $\mathrm{I}_{2}$. It is interesting to note that there is no spontaneous Au migration with spiro-MeOTAD; the reactive defects are likely neutralized by the spiro-MeOTAD prior to Au deposition. 


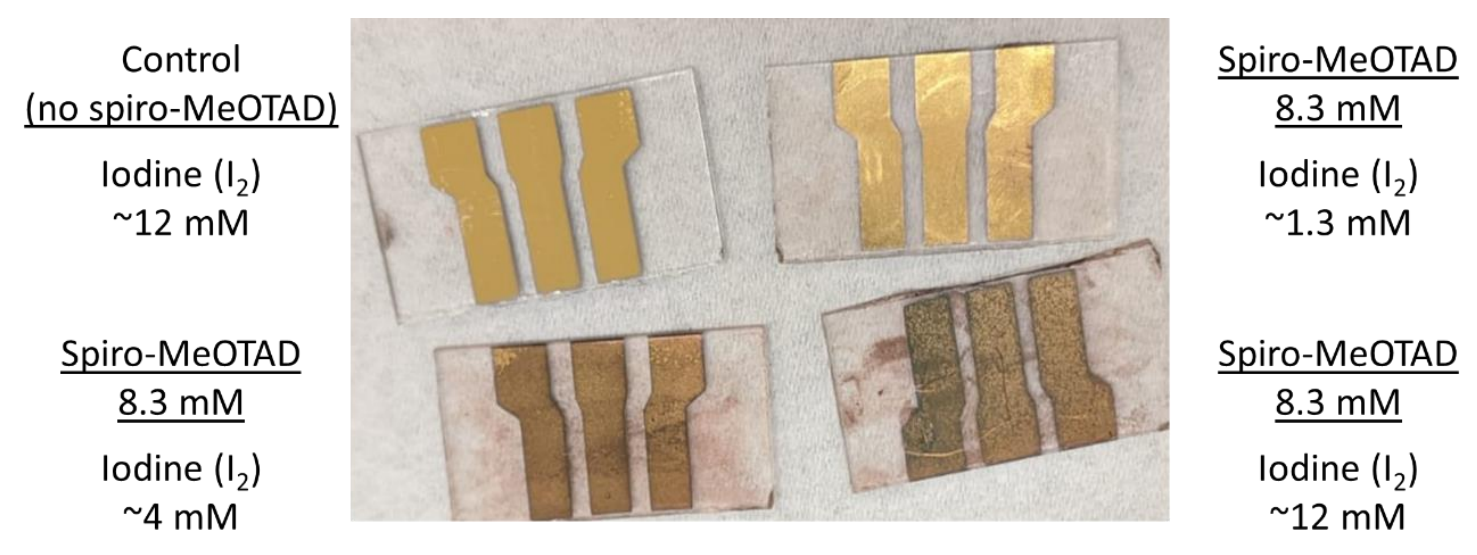

Supporting Information Figure S12. Photographs of Ti/Au thin films on glass left in solutions of spiroMeOTAD: $I_{2}$ in toluene with constant Spiro-MeOTAD content and varying amounts of $I_{2}$ for $30 \mathrm{~min}$. The Au is corroded in the simultaneous presence of Spiro-MeOTAD and $I_{2}$ due to the reaction and formation of triiodide and the rate is strongly influenced by the $I_{2}$ concentration. Molecular $I_{2}$ in toluene does not corrode Au on these time scales.

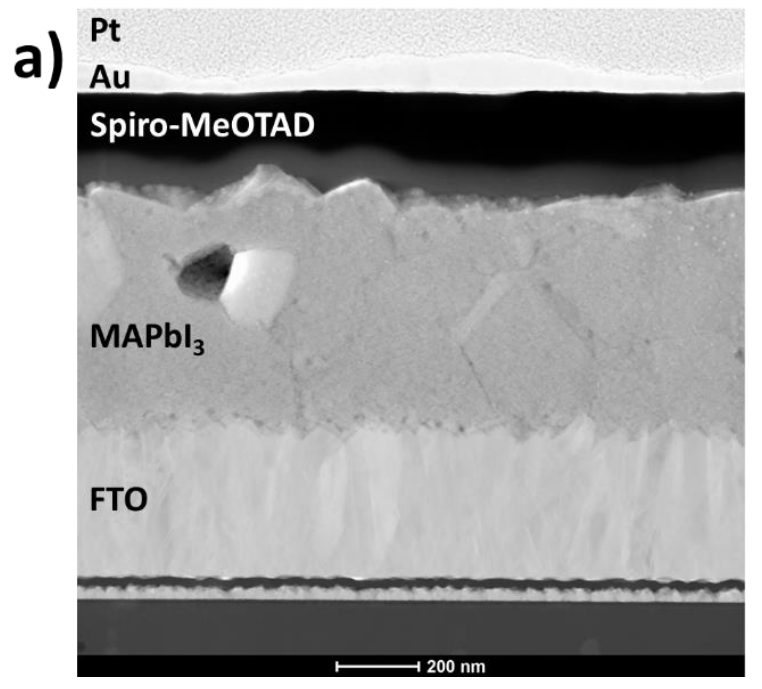

b)

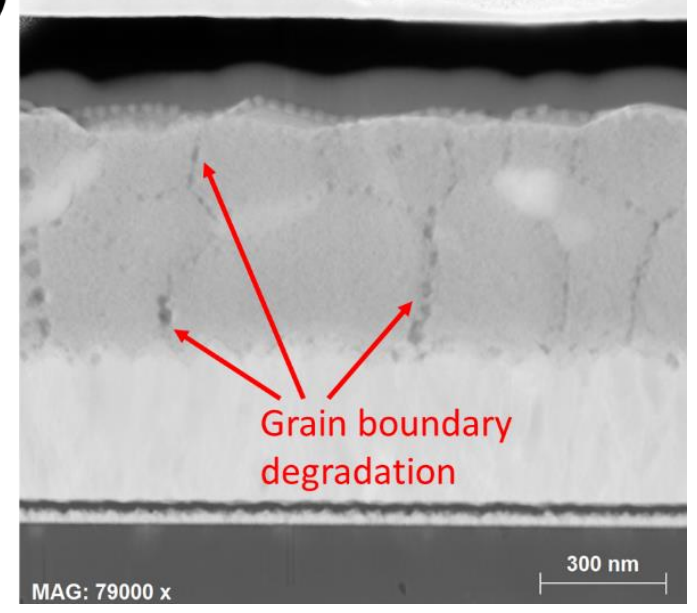

Supporting Information Figure S13. Dark-field STEM images of an FTO/MAPbl 3 /spiro-MeOTAD/Au device (a) control and (b) biased at $1.2 \mathrm{~V}$ for $16 \mathrm{~h}$. Accumulation of Au was not significant enough to resolve with TEM. However, the grain boundaries have been compromised leading to visible haziness of the intact device. The degree of degradation visible in this image closely resembles the degradation reported by Jiang et al., which together corroborate several $\mathrm{nm}$ of $\mathrm{MAPbl}_{3}$ consumption at grain boundaries. ${ }^{7}$ 


\section{References}

1. Harvey, S.P.; Li, Z.; Christians, J. A.; Zhu, K.; Luther, J. M.; Berry, J. J., Probing Perovskite Inhomogeneity beyond the Surface: TOF-SIMS Analysis of Halide Perovskite Photovoltaic Devices. ACS Appl. Mater. Interfaces, 2018, 10, 28541-28552.

2. Harvey, S. P.; Messinger, J.; Zhu, K.; Luther, J. M.; Berry, J. J., Investigating the Effects of Chemical Gradients on Performance and Reliability within Perovskite Solar Cells with TOF-SIMS. Adv. Energy Mater., 2020, 1903674.

3. Harvey, S. P.; Zhang, F.; Palmstrom, A.; Luther, J. M.; Zhu, K.; Berry, J. J., Mitigating Measurement Artifacts in TOF-SIMS Analysis of Perovskite Solar Cells. ACS Appl. Mater. Interfaces, 2019, 11, 30911-30918.

4. Yamamoto, J.; Furukawa, Y., Electronic and Vibrational Spectra of Positive Polarons and Bipolarons in Regioregular Poly(3-hexylthiophene) Doped with Ferric Chloride. J. Phys. Chem. B, 2015, 119, 4788-4794.

5. Koizumi, H.; Dougauchi, H.; Ichikawa, T., Mechanism of Dedoping Processes of Conducting Poly(3-alkylthiophenes). J. Phys. Chem. B, 2005, 109, 15288-15290.

6. Minakata, T.; Nagoya, I.; Ozaki, M., Highly ordered and conducting thin film of pentacene doped with iodine vapor. J. Appl. Phys., 1991, 69, 7354-7356.

7. Jiang, Y.; Yang, S.-C.; Jeangros, Q.; Pisoni, S.; Moser, T.; Buecheler, S.; Tiwari, A. N.; Fu, F., Mitigation of Vacuum and Illumination-Induced Degradation in Perovskite Solar Cells by Structure Engineering. Joule, 2020, 4, 1087-1103. 